\title{
Case Report \\ Reversal of a Suspected Paradoxical Reaction to Zopiclone with Flumazenil
}

\author{
Zarah Jordahn, ${ }^{1}$ Cheme Andersen, ${ }^{2}$ Anne Marie Roust Aaberg, ${ }^{3}$ and Frank Christian Pott ${ }^{3}$ \\ ${ }^{1}$ Department of Anaesthesiology, Rigshospitalet, Glostrup Matriklen, Copenhagen, Denmark \\ ${ }^{2}$ Department of Anaesthesiology, Copenhagen University Hospital, Hvidovre, Denmark \\ ${ }^{3}$ Department of Anaesthesia and Intensive Care, Bispebjerg Hospital, Copenhagen, Denmark
}

Correspondence should be addressed to Zarah Jordahn; zarahjordahn@gmail.com

Received 22 June 2016; Accepted 24 August 2016

Academic Editor: Kurt Lenz

Copyright (C) 2016 Zarah Jordahn et al. This is an open access article distributed under the Creative Commons Attribution License, which permits unrestricted use, distribution, and reproduction in any medium, provided the original work is properly cited.

\begin{abstract}
We describe the care for an elderly woman who was admitted to the intensive care unit (ICU) to receive noninvasive ventilation for acute exacerbation of chronic obstructive pulmonary disease. After administration of the sleeping pill zopiclone, a nonbenzodiazepine receptor agonist (NBRA), the patient became agitated and was confused, a possible paradoxical reaction to benzodiazepines. These symptoms were immediately resolved after treatment with flumazenil, usually used to reverse the adverse effects of benzodiazepines or NBRAs and to reverse paradoxical reactions to benzodiazepines. This case indicates that zopiclone induced behavioral changes resembling a paradoxical reaction to benzodiazepines and these symptoms may be treated with flumazenil.
\end{abstract}

\section{Introduction}

Delirium is a disturbance of consciousness and cognition that develops within hours or days. It fluctuates and appears in up to $80 \%$ of patients in the intensive care setting and has a multifactorial etiology [1]. Approaches to prevent delirium include pharmacological and nonpharmacological interventions [2]. Sleep disturbances are associated with delirium, and while the exact causal relationship is unknown, securing sleep of hospitalized patients is imperative $[3,4]$. Thus, between $41 \%$ and $96 \%$ of elderly patients admitted to the hospital are treated for sleep disturbances [5]. Drugs often used for this purpose are the so-called Z-drugs (zolpidem, zopiclone) also referred to as nonbenzodiazepine receptor agonists (NBRAs). Like benzodiazepine, Z-drugs act on the gamma-aminobutyric acid-A-receptor (GABA-A-receptor) complex, by improving sleep quality and reducing sleep latency [6]. Reports of side effects are rare but may include complex behavior such as sleepwalking and sleep eating, without any recollection of the event [7]. We describe a patient who became agitated and aggressive several hours after administration of zopiclone. This resembled delirium but may have been a paradoxical reaction to zopiclone [8].
The symptoms immediately resolved after treatment with flumazenil, usually used to reverse the adverse effects of benzodiazepines or NBRAs but can also reverse paradoxical reactions to benzodiazepines $[9,10]$.

\section{Case Presentation}

An 81-year-old woman with severe chronic obstructive pulmonary disease presented to the medical emergency department with dyspnea. She was diagnosed with acute exacerbation of chronic obstructive pulmonary disease caused by pneumonia and was treated with antibiotics, inhaled bronchodilators, systemic corticosteroids, and noninvasive ventilation. When she failed to improve, she was transferred to the intensive care unit, and to treat her sleeping difficulties dexmedetomidine $(100 \mu \mathrm{g} / \mathrm{mL})$ was infused at a rate of 8 $14 \mathrm{~mL} / \mathrm{h}$, without effect, however. On the following night, one tablet of $7.5 \mathrm{mg}$ zopiclone was given with initially good effect. After four hours of noninterrupted sleep, the patient woke up agitated, confused, and restless. Since these behavioral changes resembled a paradoxical reaction to benzodiazepine, $0.2 \mathrm{mg}$ flumazenil was given intravenously. After a few minutes, all pathological behavioral symptoms resolved and did 
not reappear during hospitalization. The patient had no recall of the episode. Previous intake of zopiclone was not registered in her medical charts, but it is unknown whether the patient had taken zopiclone before. She had no history of alcohol abuse or intake of sedatives, benzodiazepines, or antipsychotic drugs. With the exception of dexmedetomidine, no other drug suspected to interfere with the metabolism of zopiclone was given.

The patient was transferred from the ICU to the medical ward and after 3 days discharged to a temporary care center. Two days later, exacerbation in her chronic obstructive pulmonary disease resulted in readmission to the hospital, where the decision was taken to cease active care. The patient died shortly after.

\section{Discussion}

This report describes reversal of an assumed paradoxical reaction to zopiclone with flumazenil in an elderly woman. While paradox reactions and complex behavior associated with the administration of benzodiazepines are well described phenomena [8], to our knowledge, only two cases of agitation following Z-drug administration have been published $[11,12]$. In contrast, case reports on complex behavior are more abundant [7], and, in these cases, discontinuing the drug resolved the symptoms.

The mechanism behind complex behavior and paradoxical reactions remains unclear. Predisposing factors such as age, sex, concomitant disease states, psychological disturbances, and/or alcohol abuse have been proposed [7, 8]. Benzodiazepine and Z-drugs act on the GABA-A receptor, producing effects such as sedation, sleep induction, and amnesia. The GABA-A-receptor complex consists of several heterogenic subunits; the different subunits and their combination create different subtypes of the receptor. While benzodiazepines have a high affinity for several subunits, Zdrugs mostly bind to the $\alpha$-1-subunit [13].

Z-drug concentrations exceeding the recommended level have been suggested to be responsible for the occurrence of complex behaviors [7]. Whether intentional or accidental or as a consequence of drug-on-drug action, a concentration above the recommended dose may lead to decreased selectivity of Z-drug to the $\alpha$-1-subunit, thus producing effects more like those of benzodiazepines, including paradoxical reactions. Our patient received the recommended dose, but, considering her age and state of health at the time of administration, a relative overdose of the drug could be the cause of the reaction seen. Furthermore, prior to the administration of zopiclone, the patient was treated with dexmedetomidine, which, like zopiclone, is metabolized in the liver [14]. Zopiclone is metabolized by CYP 3A4 and inhibitors of cytochrome oxygenase can increase plasma concentration of this drug and therefore may induce side effects. However, no drugs that inhibit CYP 3A4, like macrolides, were given. Intoxication/overdose was not suspected, but toxicological screening was not performed. We can only speculate as to whether genetic variation in the function of the GABA receptor subunit genes had a role in developing the unexpected effects observed.
Flumazenil acts as an antagonist on the GABA-A receptor and is primarily used as antidote in benzodiazepine intoxication and benzodiazepine-related paradoxical reactions $[8,15$, 16]. Effect on Z-drug overdose and reversal of the associated adverse effects has also been reported [9, 17-19] but the exact mechanism of action of flumazenil is unknown. Flumazenil is generally well tolerated but should be used with caution in patients chronically treated with benzodiazepine and where benzodiazepines are given to control epileptic seizures.

Our patient partly fulfilled the criteria for development of delirium. A causal relationship between the use of Z-drugs and development of delirium was not demonstrated. A single test dose of flumazenil may be considered for elderly patients who develop delirium after administration of Z-drugs.

\section{Competing Interests}

The authors declare that they have no competing interests.

\section{References}

[1] S. K. Inouye, "Delirium in older persons," The New England Journal of Medicine, vol. 354, no. 11, pp. 1157-1165, 2006.

[2] I. Abraha, F. Trotta, J. M. Rimland et al., "Efficacy of nonpharmacological interventions to prevent and treat delirium in older patients: a systematic overview. the SENATOR project ONTOP series," PLoS ONE, vol. 10, no. 6, Article ID e0123090, 2015.

[3] G. L. Weinhouse, R. J. Schwab, P. L. Watson et al., "Bench-tobedside review: delirium in ICU patients-importance of sleep deprivation," Critical Care, vol. 13, no. 6, article 234, 2009.

[4] B. B. Kamdar, D. M. Needham, and N. A. Collop, "Sleep deprivation in critical illness: its role in physical and psychological recovery," Journal of Intensive Care Medicine, vol. 27, no. 2, pp. 97-111, 2012.

[5] R. Sterniczuk, B. Rusak, and K. Rockwood, "Sleep disturbance in older ICU patients," Clinical Interventions in Aging, vol. 9, pp. 969-977, 2014.

[6] C. Dolder, M. Nelson, and J. McKinsey, "Use of nonbenzodiazepine hypnotics in the elderly: are all agents the same?” CNS Drugs, vol. 21, no. 5, pp. 389-405, 2007.

[7] C. R. Dolder and M. H. Nelson, "Hypnosedative-induced complex behaviours: incidence, mechanisms and management," CNS Drugs, vol. 22, no. 12, pp. 1021-1036, 2008.

[8] C. E. Mancuso, M. G. Tanzi, and M. Gabay, "Paradoxical reactions to benzodiazepines: literature review and treatment options," Pharmacotherapy, vol. 24, no. 9, pp. 1177-1185, 2004.

[9] C.-C. Yang and J.-F. Deng, "Utility of flumazenil in zopiclone overdose," Clinical Toxicology, vol. 46, no. 9, pp. 920-921, 2008.

[10] P. W. Moore, J. W. Donovan, K. K. Burkhart et al., "Safety and efficacy of flumazenil for reversal of iatrogenic benzodiazepineassociated delirium toxicity during treatment of alcohol withdrawal, a retrospective review at one center," Journal of Medical Toxicology, vol. 10, no. 2, pp. 126-132, 2014.

[11] W. Yang, M. Dollear, and S. R. Muthukrishnan, "One rare side effect of zolpidem-sleepwalking: a case report," Archives of Physical Medicine and Rehabilitation, vol. 86, no. 6, pp. 12651266, 2005.

[12] R. D. Rattehali, S. Deshpande, and M. Jayaram, "Paradoxical agitation and sexual disinhibition following zopiclone," Prog Neurol Psychiatry-Case Notes, vol. 1, no. 1, pp. 1-4, 2009. 
[13] D. J. Nutt and S. M. Stahl, "Searching for perfect sleep: The continuing evolution of GABAA receptor modulators as hypnotics," Journal of Psychopharmacology, vol. 24, no. 11, pp. 16011612, 2010.

[14] J. Afonso and F. Reis, "Dexmedetomidine: current role in anesthesia and intensive care," Revista Brasileira de Anestesiologia, vol. 62, no. 1, pp. 118-133, 2012.

[15] B. F. Jackson, L. A. Beck, and J. D. Losek, "Successful flumazenil reversal of paradoxical reaction to midazolam in a child," The Journal of Emergency Medicine, vol. 48, no. 3, pp. e67-e72, 2015.

[16] S. R. Votey, G. M. Bosse, M. J. Bayer, and J. R. Hoffman, "Flumazenil: a new benzodiazepine antagonist," Annals of Emergency Medicine, vol. 20, no. 2, pp. 181-188, 1991.

[17] Z. Ahmad, M. Herepath, and P. Ebden, "Diagnostic utility of flumazenil in coma with suspected poisoning," British Medical Journal, vol. 302, no. 6771, p. 292, 1991.

[18] S. L. Thornton, E. Negus, and S. D. Carstairs, "Pediatric zolpidem ingestion demonstrating zero-order kinetics treated with flumazenil," Pediatric Emergency Care, vol. 29, no. 11, pp. 1204-1206, 2013.

[19] J. J. Cienki, K. K. Burkhart, and J. W. Donovan, "Zopiclone overdose responsive to flumazenil," Clinical Toxicology, vol. 43, no. 5, pp. 385-386, 2005. 


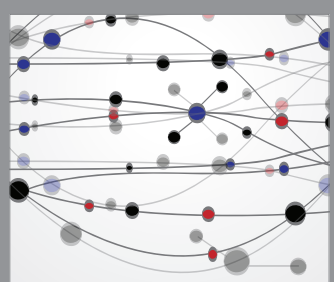

The Scientific World Journal
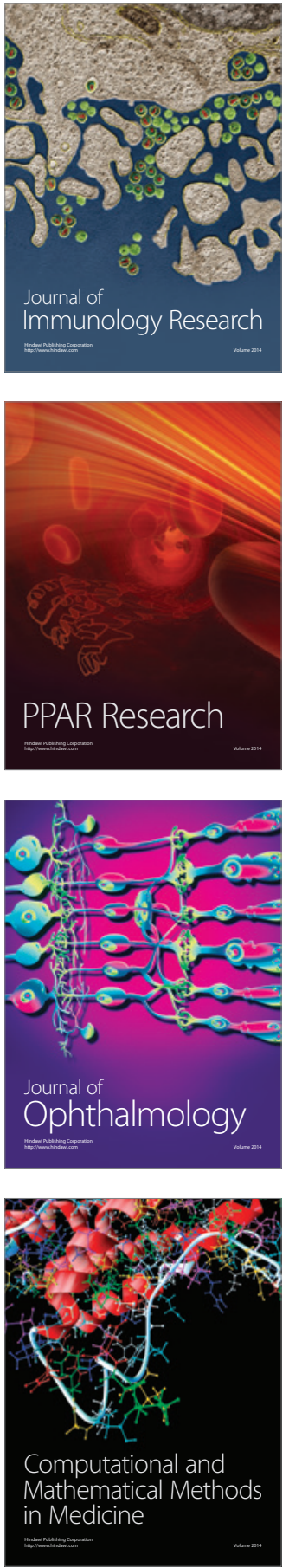

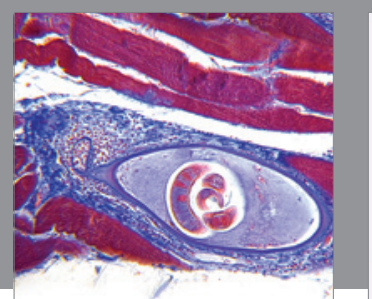

Gastroenterology Research and Practice

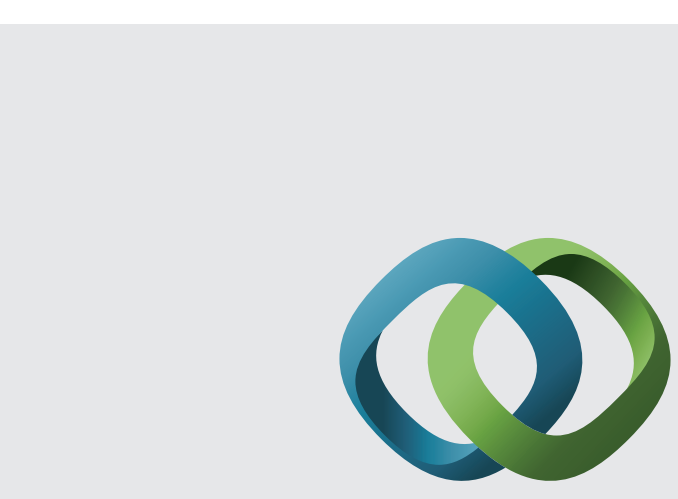

\section{Hindawi}

Submit your manuscripts at

http://www.hindawi.com
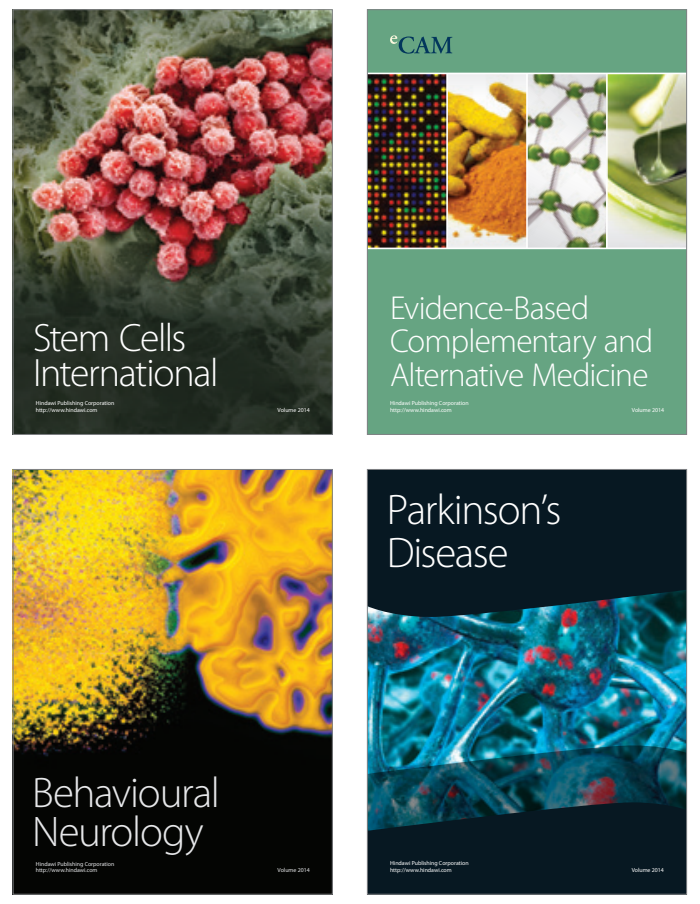
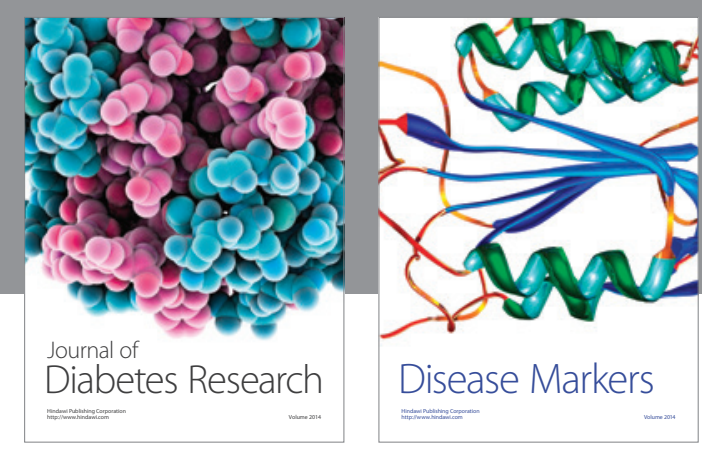

Disease Markers
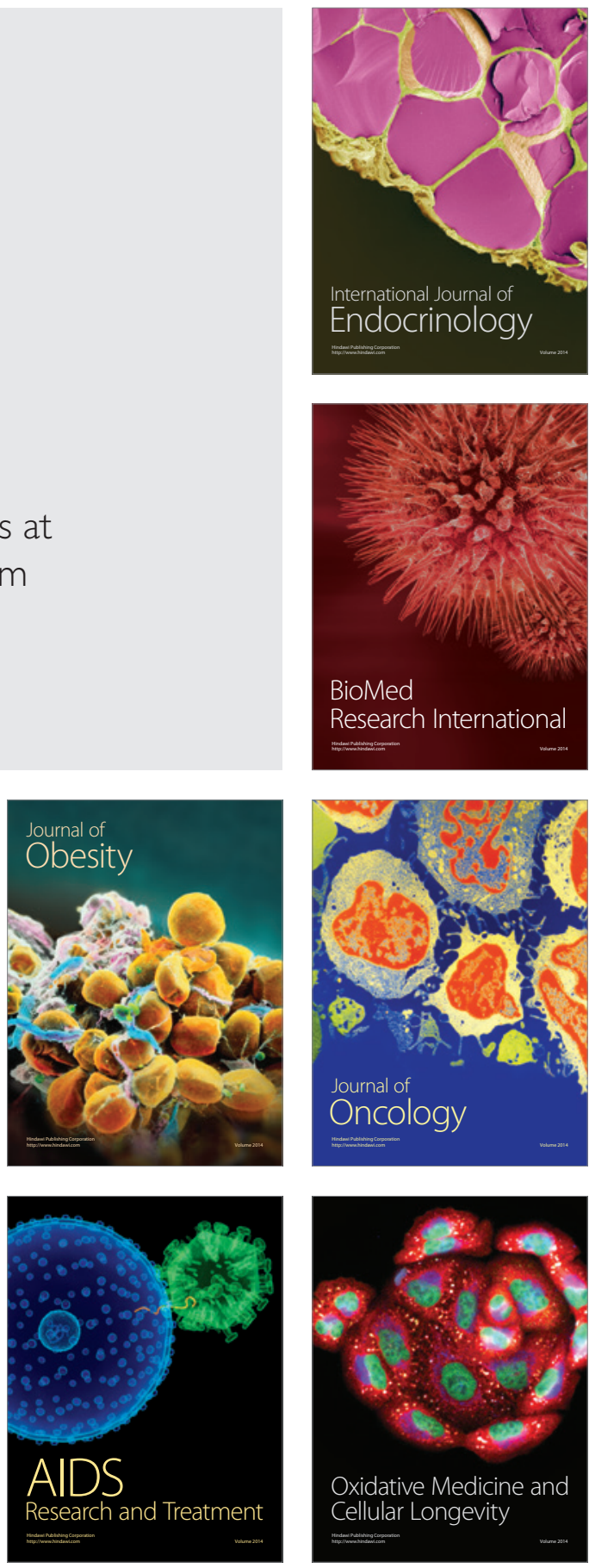